\title{
Jumper's fracture of the sacrum: a novel and reproducible way for successful reduction and fixation
}

\author{
Christoph Linhart ${ }^{1} \cdot$ Nima Befrui $^{1} \cdot$ Lucas Etzel $^{2} \cdot$ Wolfgang Böcker $^{1} \cdot$ Christian Kammerlander $^{1}$. \\ Christian Ehrnthaller ${ }^{1}$
}

Received: 27 November 2020 / Revised: 15 September 2021 / Accepted: 22 September 2021 / Published online: 5 October 2021

(c) The Author(s) 2021

\begin{abstract}
Purpose Surgical treatment of jumper's fractures is a highly demanding situation for the surgeon due to its rareness and frequent association with severe concomitant injuries. There is no current consensus regarding a standard treatment approach, thus reducing quality of care. Our objectives were to describe, apply and assess a novel surgical technic.

Methods The presented research is an observational retrospective study of patients who underwent the described novel surgical intervention in a level 1 trauma center. We conducted analyses of the patient cohort using patient-related outcome measures at least 1 year after surgery, as well as investigating pain, quality of life and the clinical effectiveness of the procedure. Results A total of 24 patients ( 17 male and 7 female) with an average age $47 \pm 16.3$ years were included. ISS scores ranged from 9 to 66 with a mean ISS of $40 \pm 15$. Clinical scores exist of 15 out of 24 patients (62.5\%). The mean VAS score was $53.7 \pm 12.9$. The mean EQ-5D index was $0.68 \pm 0.22$. Significant negative correlation existed between the ISS value and the EQ-5D index $(r=-0.704 ; p<0.005)$ and EQ-5D VAS $(r=-0.809 ; p<0.001)$. Anatomical reduction was achieved in all patients $(n=24)$. Radiological follow-up was performed in $58 \%$.

Conclusion We present one of the largest studies with operatively treated jumper's fractures of the sacrum. The technique is capable of reproducibly restoring the physiological anatomy of the patient and allows pain-adapted mobilization.
\end{abstract}

Keywords Spine $\cdot$ Jumper's fracture $\cdot$ Sacral fracture $\cdot$ Reduction $\cdot$ Lumbosacral spine $\cdot$ Technique $\cdot$ Surgical treatment $\cdot$ Dorsal instrumentation

\section{Introduction}

The horizontal sacral burst fracture, also known as jumper's fracture, describes an anterior dislocation of the spine in relation to the pelvic plane. In the classification according to Roy-Camille, it is a type 3 or 4 fracture, and the Association for the Study of Osteosynthesis (AO) uses the designation AO/OTA C3 for this type of fractures [1]. Most commonly,

Christian Kammerlander and Christian Ehrnthaller have contributed equally to the study and shared last authorship.

Christoph Linhart

christoph.linhart@med.uni-muenchen.de

1 Department of General, Trauma and Reconstructive Surgery, University Hospital, LMU Munich, Campus Großhadern, Marchioninistr. 15, 81377 Munich, Germany

2 Division of Hand, Plastic and Aesthetic Surgery, University Hospital, LMU Munich, Munich, Germany jumper's fractures occur after a fall from great height with high axial force applied to the body. Due to the severity and high velocity character of the injury, neurological symptoms as well as additional injuries are frequent.

There are three major issues which make this injury entity demanding and challenging for the surgeon on call: First, due to the rarity of this type of injury, only few surgeons possess great expertise in the treatment of these injuries. Second, as neurological symptoms are common, jumper's fractures should be treated as early as possible, making it often impossible to postpone the surgical intervention to the "best time, best team" timeframe. And third, until now, there is no gold standard or standard approach of treating these injuries, which reduces the quality of care, as reduction is challenging and based upon a reproducible reduction technique.

Currently, there are only a few case reports which deals with a reproducible fixation technique and all describe different reduction maneuvers to correct the malposition [2-4]. 
In comparison, to the authors' knowledge, there is only one study with a greater patient collective in which spinopelvic dissociation is investigated, whereby the focus of this work is not primarily on the surgical technique itself [5].

The first described ways of treating these injuries mainly incorporated sole fixation of the often-displaced fractures by iliac bars, posterior plating or classical dorsal internal fixation devices. Unfortunately, fracture reduction was barely achievable due to technical impediments. As surgical management by use of the aforementioned techniques was impaired, the complication rates were high and the functional outcome limited [1, 6-11].

Many of these authors also proposed sacro-iliac fusion as the treatment option of choice. This technique can be performed minimally invasive and therefore represents a "mild" way of treatment for these often critically injured patients. But only recently, other studies demonstrated that pelvic incidence increases after sacro-iliac fusion possibly due to increased rotational instability [12]. Ultimately, this increased rotational instability may result in increased lumbar lordosis affecting spondylarthrosis [13].

As mentioned in the technical recommendations by Piltz et al. [2], one of the key difficulties in treating these injuries is the problem with implant failure, as high leverage forces act upon the fixation implants. The authors therefore described a technique where reduction is possible through combination of restoring length via rod distraction and lordosis by using a hinge mechanism between the longitudinal rods and a horizontal rod between both posterior superior iliac processes.

In comparison with the aforementioned technique, we used a modification of the technique described by Staranzis et al. in 2014 [3]. Here, solid rods are used instead of a hinge mechanism in order to maintain reduction and fixation of these unstable injuries. The technique is based on one of the most frequently used implants for the spine, the universal spine system (USSII) from DePuy Synthes, which makes it highly reproducible for interested surgeons. Thus, reduction is performed via its specific surgical instruments, namely the distraction forceps which is used to restore longitudinal length between the pedicle screws of L4 and L5, and the "persuader", which is used to restore lordosis and dislocation of the spine in anterior direction.

The hypothesis of the study was that an increase in stability due to the omission of the hinge mechanism and the resulting low-profile construction would lead to a better clinical outcome. Therefore, we analyzed our patients regarding patient related outcome measures (PROMs) and clinical effectiveness of our surgical procedure.

\section{Materials and methods}

\section{Structure of the study}

This study was approved by our institutional review board with the approval no. 18-745.

The presented research is an observational study of 24 patients who underwent the above-mentioned surgical intervention between January 2015 and December 2019 in a level 1 trauma center in Germany. The study included patients between the ages of 18 and 99 years who had suffered a fracture due to a high-level accident. Patients with fractures caused by an infection, tumor of the spine or osteoporotic fragility fractures of the pelvis were excluded from the study.

The following data were collected for each patient after analyzing the emergency admission reports, anesthesia protocols, surgery reports and physician letters:

- Age, sex

- Fracture localization and classification

- History of fracture

- ASA Score

- Injury Severity Score (ISS)

- Surgical procedure(s), duration of surgery

- Intraoperative blood loss as well as intraoperative complications

- Duration of stay in intensive care unit (ICU)

- Length of hospital stay (LOS), concomitant injuries

- radiological imaging postoperative -6 weeks -3 months -6 months -1 year and removal of implanted material.

The AO-Classification was used to classify the fractures. Clinical follow-up with radiographic evaluation of patients was performed at the regular timepoints of 6 weeks, 3 months, 6 months and 1 year post-op. PROMs were collected in a long-term follow-up phase, which we defined as ranging up to 5 years after patient discharge (mean: $28 \pm 19$ months after patient discharge).

For standardized evaluation of postoperative healthrelated quality of life, we used the Core Outcome Measurement Index (COMI) and the Oswestry Low Back Pain Disability Index 2.1 (ODI), both of which are self-administered and multidimensional Patient-Reported Outcome Measures (PROMs). The EuroQol EQ-5D questionnaire was used for generic assessment of postoperative quality of life, while the Visual Analogue Scale (VAS) was used to measure the pain situation. The descriptive measures used were mean, median, standard deviation (SD) and range, calculated with SPSS Statistics Version 25 (IBM, USA). 


\section{Surgical technique}

The applied technique is a modification of the procedure used in the study by Staranzis et al. [3]. The lumbosacral transition and laminectomy of the sacrum is performed in a prone position with inflected hip and knee joints. As a first step, the fourth lumbar vertebra is pediculated using the polyaxial USSII system screw (DePuy Synthes USSII iliosacral/polyaxial). In the next step, the superior posterior iliac process is osteotomized in an inclined osteotomy plane tilted toward the midline to minimize the soft tissue irritation by the implants and achieve the lowest possible implant profile. The ileum screws are inserted at a $45^{\circ}$ angle laterally and a $30^{\circ}$ angle distally to obtain a maximum running length of the screws in the os ilium. An additional iliac connector is now inserted and reduces the height of the implant construct even further. Subsequently, slightly pre-bent longitudinal rods are inserted, and the reduction of the spinal column to the os sacrum is performed by gradual longitudinal traction between the L4 and os ilium screws with the reduction instrument and intermittent locking of the longitudinal rod. By restoring the length of the spinal column, the lumbar vertebra 5 comes into view, making its pediculation possible. Analog to the fourth lumbar vertebra, polyaxial screws are inserted. The translation in the sagittal plane is now corrected by maneuvering the longitudinal rod into the pedicle screw L5 with the aid of the system specific USSII persuader. This maneuver is performed alternately on both sides until the normal anatomy is restored. Finally, a transverse connector is inserted to increase stability.

Immediate full weight-bearing was allowed without further restrictions (Figs. 1 and 2).

\section{Results}

24 patients who had undergone iliolumbar stabilization of a jumper's fracture of the sacrum were included in this study. This patient cohort included 17 male and 7 female patients with ages ranging from 21 to 80 years. The average age was $47 \pm 16.3$ years.

The most common mechanism of injury was a fall from a height greater than $3 \mathrm{~m}(50.0 \%, n=12)$, followed by high impact collisions usually involving vehicles in road traffic accidents $(33.3 \%, n=8) .8 .3 \%(n=2)$ of cases sustained their injuries in falls from a height of less than $3 \mathrm{~m}$. In 2 cases (8.3\%), the trauma mechanism could not be determined due to incomplete information from first responders and/ or the patient, e.g., in case of psychotic episodes (Figs. 3 and 4).

The patient's ASA classification ranged from II (20.8\%, $n=5)$ to IV $(29.2 \%, n=7)$, with ASA III being the most
Fig. 1 Photographs of the applied lumbopelvic instrumentation and reduction technique. $\mathbf{a}=$ Preoperative status with complete dissociation of the spine to the pelvis. $\mathbf{b}=$ Pediculation of L4. $\mathbf{c}=$ Osteotomy of the superior posterior iliac process and insertion of iliac screws. $\mathbf{d}=$ Restoration of length via distraction forceps between screws L4 and Os ilium. $\mathbf{e}=$ Restoration of anterior translation of the spine with the persuader after insertion of L5 screws. $\mathbf{f}=$ Final construct
A

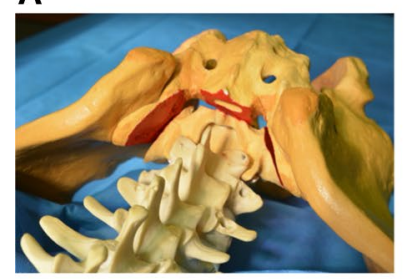

D

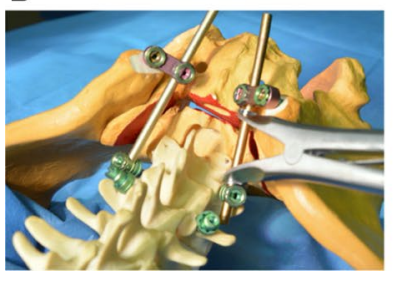

B

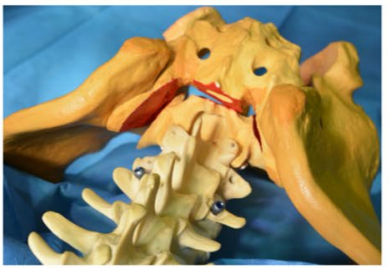

E

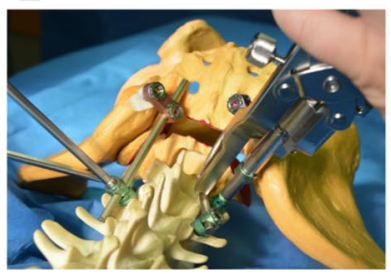

C

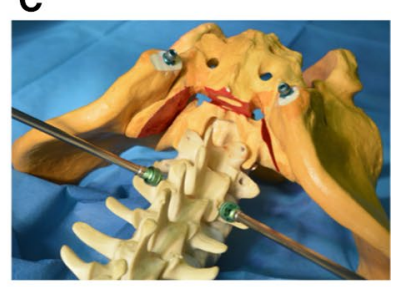

$\mathbf{F}$

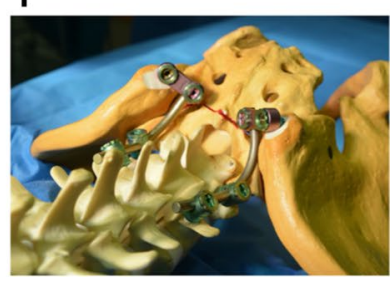

Fig. 2 Three-dimensional image of the anatomical situation with dislocated spine in respect to the pelvic bone (a) and after reduction (b). The fracture lines are indicated in red
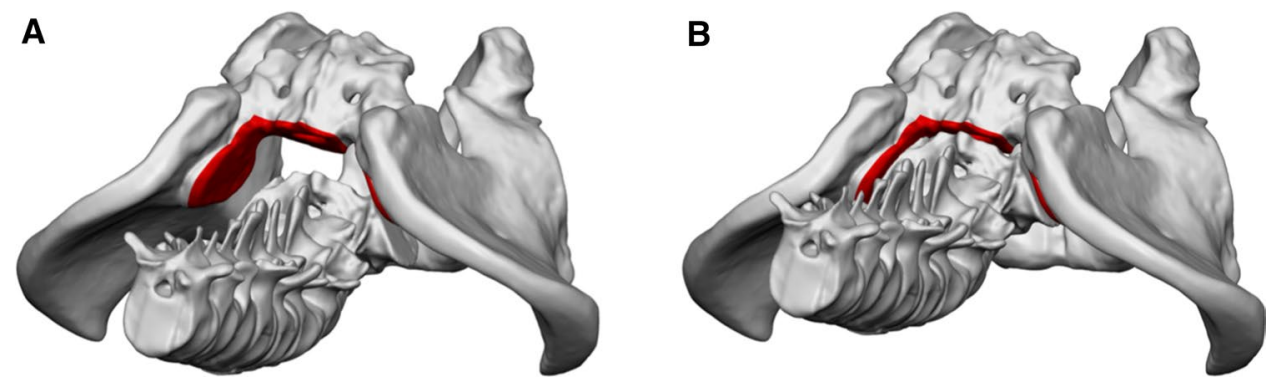
Fig. 3 Pre- and postoperative computer tomography of a suicidal jumper's fracture with complete anterior dislocation of the spine in respect to the pelvis. $\mathbf{a}=$ Image before surgery. $\mathbf{b}=$ Postoperative computed tomography showing anatomical reduction with the described reduction and fixation technique
A

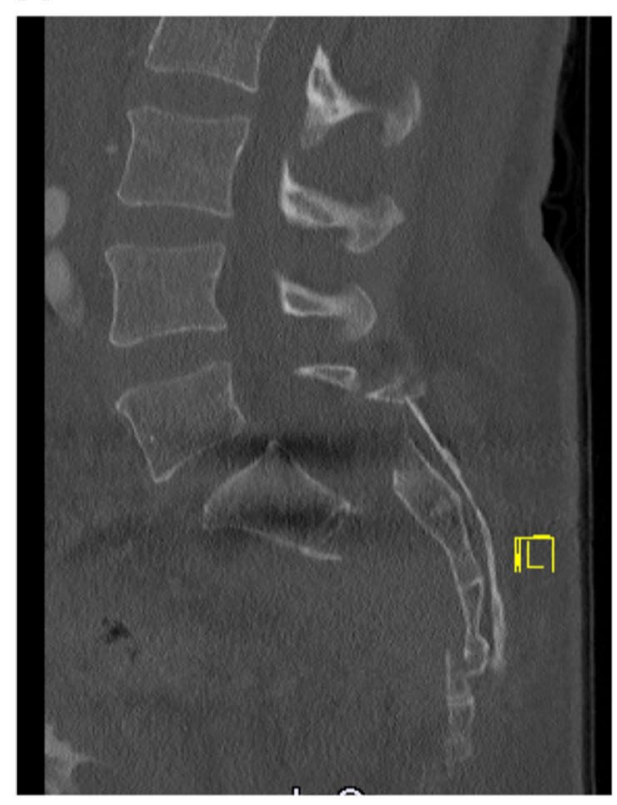

B

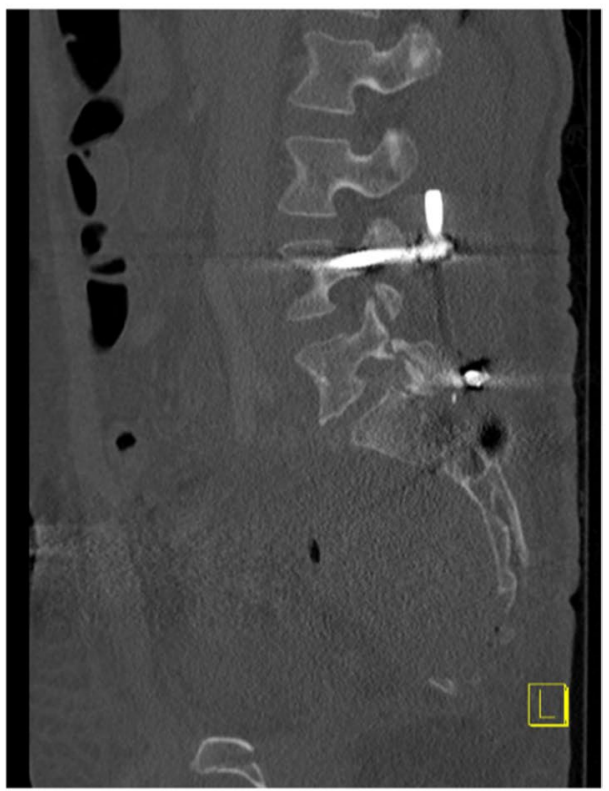

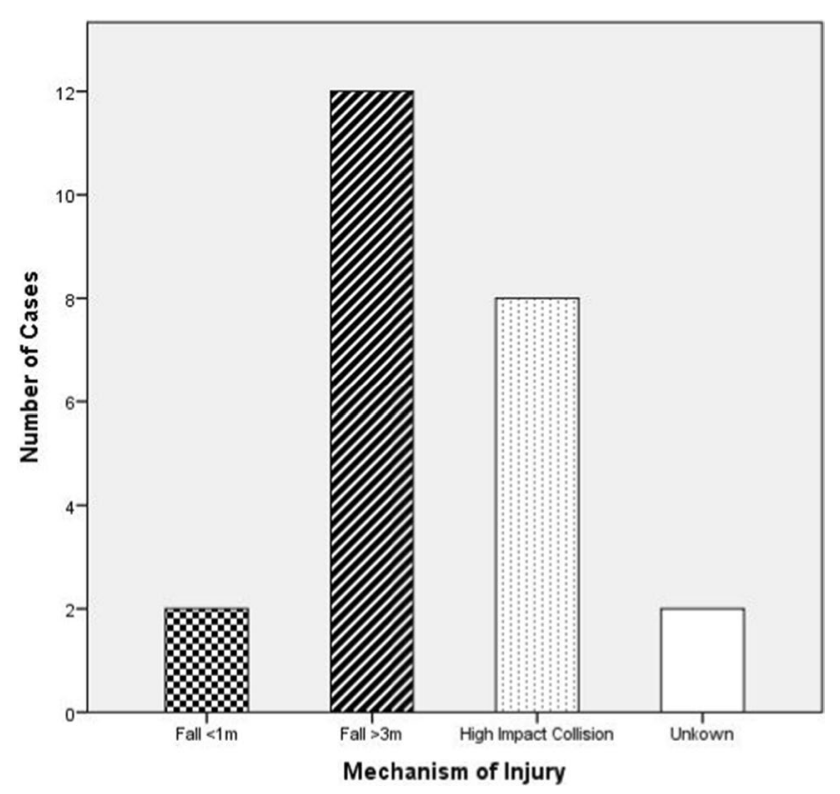

Fig. 4 Mechanisms of injury determined in the 24 sacral fracture cases. In 2 cases, the nature of the trauma could not be determined due to incomplete information from first responders and/ or the patient

frequent with $50.0 \%(n=12)$. ISS scores ranged from 9 to 66 with a mean ISS of $40 \pm 15$.

The most prevalent fracture type diagnosed in the 24 patients according to AO-Classification was a $\mathrm{C} 3$ type fracture $(83.3 \%, n=20)$. All but a single patient, who sustained a type $\mathrm{B} 3$ fracture, were diagnosed with $\mathrm{C}$ type fractures of the pelvis.
Thirteen of the 24 patients (54.2\%) sustained critical accompanying injuries of the small pelvis and/or abdomen, such as arterial bleedings with possible hemorrhagic shock, or injuries to organs, such as lacerations of the liver, spleen, kidneys and bladder.

8 of the 24 patients (33.3\%) presented neurological symptoms or deficits: 7 patients presented combinations of sensory and motor deficits, as well as voiding dysfunction and/ or incontinence. Of these 7 patients, 1 patient presented a complete spinal cord injury. 1 patient reported an isolated hypesthesia of the lower extremities (for detailed information see Table 1). In the short-term interval, no change in neurological status was observed postoperatively in the neurologically impaired patients. On trend, sensitive deficits were already declining and were documented accordingly at discharge. In the long-term interval, a definite statement is not yet possible due to the inconsistent information in the follow-up examinations.

Definitive surgical treatment of the sacral fractures was performed on average $5 \pm 4$ days (range: 0-16 days) after admittance of the patient.

In 15 of the 24 surgical cases, the operation included further procedures beyond the iliolumbar stabilization. The mean duration of the 24 surgical cases was $226 \pm 81 \mathrm{~min}$, and the blood loss ranged from 250 to $3250 \mathrm{ml}$ with a mean of $1210 \pm 861 \mathrm{ml}$.

When examining the 9 cases in which only iliolumbar stabilization was performed, the mean surgical duration was $189 \pm 38$ min with an average blood loss of $961 \pm 649 \mathrm{ml}$ (Fig. 5).

18 of the 24 patients required intensive care treatment for an average of $18.3 \pm 10.0$ days (range: $1-35$ days). Total 
Table 1 Overview of the patients included in this study, providing their respective case number, age, sex, AO-Classification of the sacral fracture, ASA, ISS scores and neurological deficits

\begin{tabular}{|c|c|c|c|c|c|c|}
\hline Case number & Age & Sex & $\begin{array}{l}\text { Fracture class. } \\
(\mathrm{AO})\end{array}$ & ASA & ISS & $\begin{array}{l}\text { Neurological } \\
\text { deficit (Type) }\end{array}$ \\
\hline 1 & 68 & $\mathrm{~m}$ & B3 & 2 & 27 & N1 \\
\hline 2 & 45 & $\mathrm{f}$ & $\mathrm{C} 3$ & 2 & 14 & No \\
\hline 3 & 46 & $\mathrm{~m}$ & $\mathrm{C} 2$ & 3 & 50 & NO \\
\hline 4 & 25 & $\mathrm{~m}$ & C3 & 2 & 41 & N0 \\
\hline 5 & 80 & $\mathrm{~m}$ & $\mathrm{C} 3$ & 3 & 9 & No \\
\hline 6 & 54 & $\mathrm{f}$ & C3 & 4 & 46 & N0 \\
\hline 7 & 44 & f & C3 & 3 & 50 & N3 \\
\hline 8 & 49 & $\mathrm{~m}$ & C3 & 3 & 22 & N3 \\
\hline 9 & 55 & $\mathrm{~m}$ & $\mathrm{C} 2$ & 4 & 50 & No \\
\hline 10 & 74 & $\mathrm{f}$ & $\mathrm{C} 3$ & 4 & 34 & No \\
\hline 11 & 25 & $\mathrm{f}$ & $\mathrm{C} 3$ & 3 & 48 & No \\
\hline 12 & 50 & $\mathrm{~m}$ & $\mathrm{C} 3$ & 2 & 34 & No \\
\hline 13 & 57 & $\mathrm{~m}$ & $\mathrm{C} 3$ & 3 & 18 & No \\
\hline 14 & 61 & $\mathrm{~m}$ & $\mathrm{C} 3$ & 3 & 48 & No \\
\hline 15 & 25 & $\mathrm{~m}$ & C3 & 4 & 50 & N4 \\
\hline 16 & 27 & $\mathrm{~m}$ & $\mathrm{C} 3$ & 3 & 48 & No \\
\hline 17 & 35 & $\mathrm{~m}$ & $\mathrm{C} 3$ & 3 & 27 & N3 \\
\hline 18 & 21 & $\mathrm{~m}$ & $\mathrm{C} 1$ & 2 & 48 & No \\
\hline 19 & 37 & $\mathrm{f}$ & $\mathrm{C} 3$ & 4 & 57 & No \\
\hline 20 & 43 & $\mathrm{~m}$ & C3 & 4 & 48 & N3 \\
\hline 21 & 64 & $\mathrm{~m}$ & C3 & 3 & 41 & N2 \\
\hline 22 & 54 & $\mathrm{f}$ & C3 & 3 & 26 & No \\
\hline 23 & 32 & $\mathrm{~m}$ & C3 & 4 & 66 & $\mathrm{~N} 2$ \\
\hline 24 & 57 & $\mathrm{~m}$ & C3 & 3 & 50 & N0 \\
\hline
\end{tabular}

Fig. 5 Intraoperative blood loss and length of surgery as determined in the case of isolated iliopelvic stabilization procedures (white box) or operations including further procedures beyond the iliopelvic stabilization (striped box)
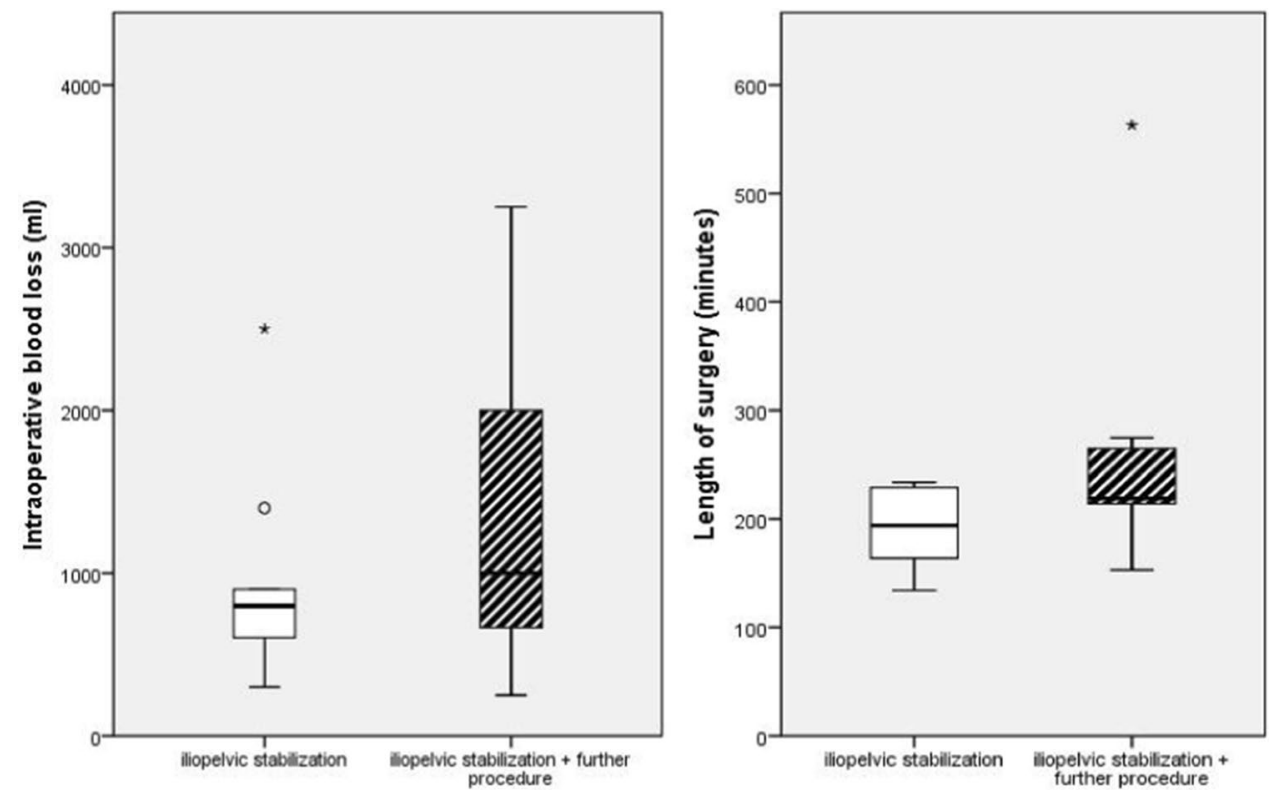

length of stay ranged from 12 to 107 days, with a mean LOS of $35 \pm 23$ days.

In 2 patients (8.3\%), intra-/or perioperative surgical complications occurred: One patient developed respiratory deficiency immediately postoperatively, requiring intensive care treatment. In the second patient, a bleeding stemming from the dorsal sacral area occurred. 
Another 2 patients (8.3\%) suffered from postoperative complications: One patient sustained a pulmonary embolism, whereas another patient developed a pneumonia and UTI.

$50 \%$ of patients presented a mental illness and/or required psychiatric treatment during or after the hospitalization.

15 of the 24 patients reported on their well-being using clinical scores in the long-term follow-up. In the VAS-score (Visual Analogue Scale) ranging from 0 (most intense pain imaginable) to 100 (no pain at all), scores ranged from 30 to 70 . The mean VAS-score was $53.7 \pm 12.9$. On the Oswestry Disability Index (ODI, higher percentages indicate greater disability), scores ranged from 18 to $48 \%$ with a mean ODIscore of $33.4 \pm 10.3 \%$. The COMI-scores, whose range spans from 0 (best possible score) to 10 (worst possible score), ranged from 2.9 to 7.9 with a mean score of $4.98 \pm 1.78$. On the EQ-5D-5L score, the EQ-5D index ranged from 0.18 to 0.89 (mean: $0.68 \pm 0.22$ ), while the EQ-5D VAS ranged from 0.18 to 0.90 (mean: $0.59 \pm 0.21$ ).

Given the above-mentioned range of ISS values of 9-66, we hypothesized that higher ISS values indicating a greater severity and/or complexity of injuries could correlate with a worse self-reported outcome after discharge. Indeed, we found a significant negative correlation between the ISS value and the EQ-5D index $(r=-0.704 ; p<0.005)$ and EQ-5D VAS $(r=-0.809 ; p<0.001)$ test results. A linear regression analysis for ISS and EQ-5D index values produced an $R^{2}$ of 0.496 (adjusted $R^{2}=0.457 ; \beta=-0.704$; $p<0.005)$. Likewise, a linear regression analysis for ISS and EQ-5D VAS values produced an $R^{2}$ of 0.654 (adjusted $\left.R^{2}=0.627 ; \beta=-0.809 ; p<0.001\right)$.

Restoration of the physiological load and axes, which we would define as an anatomical reduction and main criteria for a successful operative technique, was achieved in all of our patients $(n=24)$. This is reflected by the high implant removal rate $(n=9)$ and fusion rate $(n=5)$ patients with pending implant removal. No screw loosening or screw pull-out was observed in follow-up imaging. Radiological imaging was performed postoperative, 6 weeks, 3 months, 6 months and 1 year after the operation. Complete imaging was available in 14 cases (58\%). Balanced radiographs, skeletal alignment evaluation by upright radiographic imaging, were not in all cases performed.

\section{Discussion}

Here, we present one of the largest studies with operatively treated jumper's fractures of the sacrum.

Despite the severity of our patients' injuries, the presented reduction and fixation technique led to physiological restoration of the anatomy, a good functional outcome, and acceptable quality of life.
Until now, only individual studies with single digit number of patients were available which deals with the presentation of a reproducible fixation technique. In 2014, the working group around Lindahl et al. published a study with a collective of 36 included patients described factors associated with outcome of spinopelvic dissociation treated with lumbopelvic fixation and not primarily on the surgical technique like our presented study deals with [5].

The largest study collective which presented a reproducible surgical technique was presented by Piltz et al., where 3 patients were operated with a specific iliopelvic stabilization technique. Restoration of the anatomy was achieved via connection bars and a hinge mechanism. Using this technique, the authors were able to achieve good functional results and full mobilization of the patients. Despite these promising results, it is tempting to speculate that the use of the hinge mechanism has some disadvantages regarding stability and soft tissue irritation.

By omitting a hinge mechanism, we created a more stable construct, as a solid rod will be less likely prone to loss of reduction compared to a hinge joint created by adaption of longitudinal and horizontal rods with a certain torque.

Another key advantage of the technique presented here is the low-profile instrumentation of our construct with decreased likeliness of wound healing complications [7, 9].

Multiple studies already demonstrated that wound healing complications are a major issue in treating these injuries. Although the traumatic injury itself contributes to soft-tissue and muscle damage, which itself increases the likelihood of wound healing complications, in most of these cases it is not possible to delay surgical treatment due to massive dislocation and neurological symptoms. Besides soft-tissue damage, smear infections pose a considerable additional risk.

Besides basic hygienic principles, prophylactic treatment is limited. Although various studies examining the use of tissue adhesive as single or adjunct to conventional wound closure exist, there is no clear set of data proving the effectiveness of this procedure toward prevention of surgical site infection (SSI) [14].

The only possibility for the surgeon to contribute to this issue is to make the osteosynthesis construct as low profile as possible. For this reason, the superior posterior iliac process is osteotomized in an inclined osteotomy plane tilted toward the midline to minimize the soft tissue irritation by the implants and achieve the lowest possible implant profile.

This should be able to sufficiently reduce wound healing complications in this high-risk patient collective. Additionally, omitting the horizontal rod between both posterior superior iliac processes reduces the height of the implant in general, making wound healing complications less likely.

Another important but often neglected factor in the prevention of secondary postoperative complications is the postoperative mobilization regime. 
Earlier studies dealing with this kind of injury proposed a non-weight bearing regime for 6 weeks or a staged protocol with initial mobilization under the aid of a tilt table, followed by careful strict erect walking with a wheeled walker and avoidance of forward movement of the trunk in the first 8-12 weeks under additional lumbar orthosis [2, 10].

The latter regime might be the consequence of the low stability of the construct with the use of a hinge joint and the intention to avoid leverage forces upon the construct before bony healing increased the overall stability of the construct. Besides this technique's specific limitation, the aim for these critically injured patients should be freefunctional early mobilization and immediate full weightbearing, which is why pain-adapted free mobilization during the postoperative phase was applied to our patients.

The importance of early mobilization was only recently highlighted in a series of studies from our group. Beyond showing that partial weight-bearing was not only hard to accomplish by a lot of patients [15], it was also concluded that reduced weight bearing and inactivity throughout the first postoperative phase resulted in a critical disadvantage for these patients [16].

Therefore, the optimal treatment regime not only requires an osteosynthesis technique capable of achieving and maintaining reduction in the operating room, but also during the rehabilitation process enabling the patient an early free-functional pain-adapted full weight-bearing.

Therefore, in the present study, we allowed the patients a full range of motion with immediate full weight-bearing postoperatively.

A multitude of patients analyzed in our study presented complex injury patterns and a high rate of injury-related secondary complications. Despite the severity of these injuries, our study collective presented a good functional restoration as documented in the ODI score. In comparison, the reported quality of life was rated being in a medium range. This fact might be due to specific comorbidities of our patient cohort. As presented in Fig. 6, a majority of patients suffered from different mental health disorders, which may have influenced the self-reported quality of life assessment (Figs. 7 and 8).

As the majority of patients suffered from additional injuries, it is not possible to study the effect of the presented reduction and fixation technique due to the jumper's

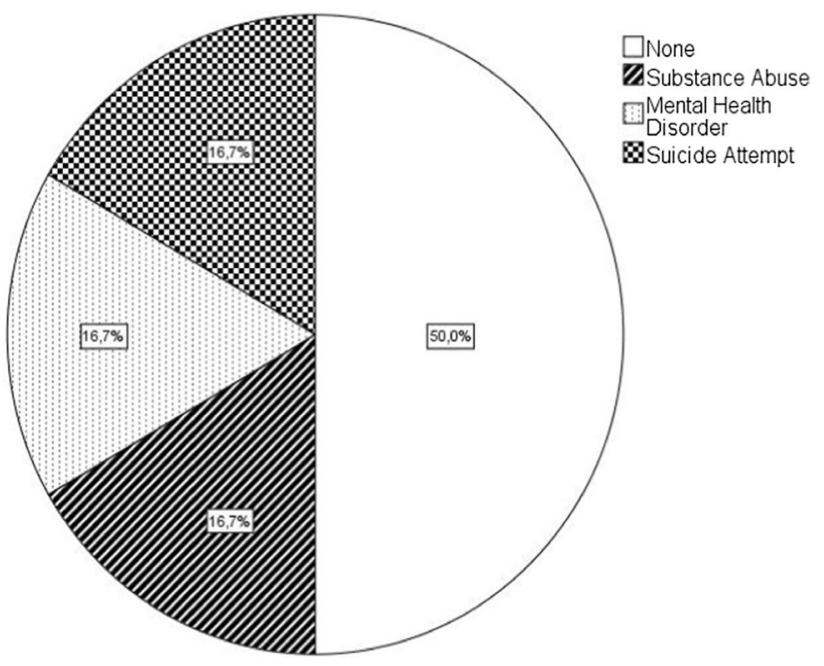

Fig. 6 Prevalence of psychiatric disorders identified in the sacral fracture patients or associated with the mechanism of injury

fracture alone. Therefore, the measured PROMs always represent the sum of all subsequent functional impairments (COMI) and pain (VAS). This is underlined by the significant correlation of the ISS with the COMI and VAS. Therefore, higher values in COMI and VAS were expected, do not represent a perfect measurement tool for analyzation of our surgical treatment and should be interpreted with care.

\section{Conclusion}

Surgical treatment of the suicidal jumper's fracture is a highly demanding situation for the surgeon due to its rareness and frequent association with severe concomitant injuries. It is therefore essential that the surgeon's repertoire includes a reliable technique for the reduction and fixation of these unstable injuries. The presented technique for reduction and fixation represents a highly reproducible approach to treating these rare and severe injuries. While making use of a widespread implant system that most spine surgeons are aware of, the technique is capable of restoring the physiological anatomy of the patient and is strong enough to allow pain-adapted mobilization in the postoperative rehabilitation phase. 
Fig. 7 VAS (a), COMI (b), ODI (c) and EQ-5D (d) scores as reported by 15 patients
A

Worst pain imaginable

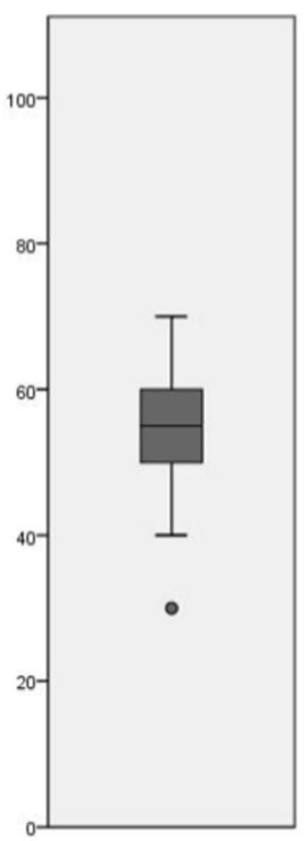

C

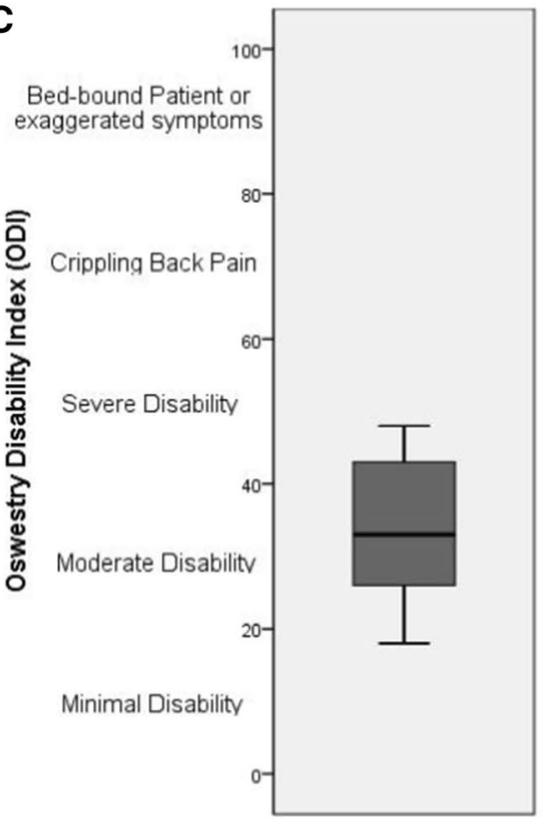

B

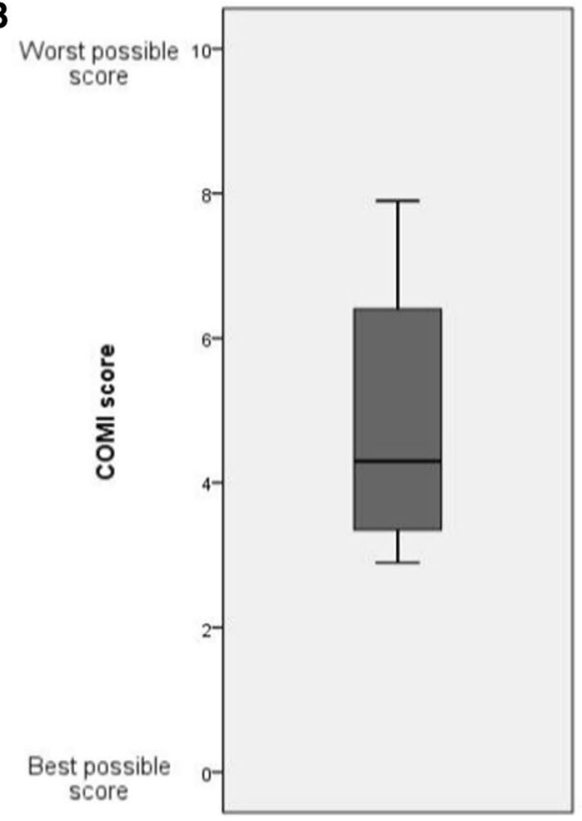

D

Best possible score

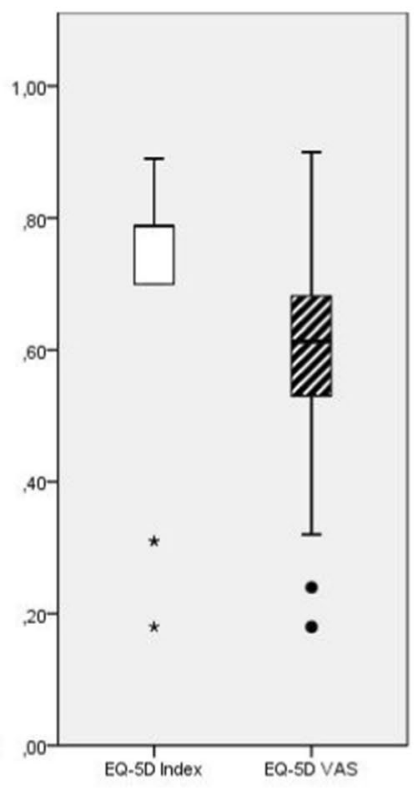


Fig. 8 Linear regression analysis for ISS and EQ-5D index (a) and EQ-5D VAS (b) scores, respectively
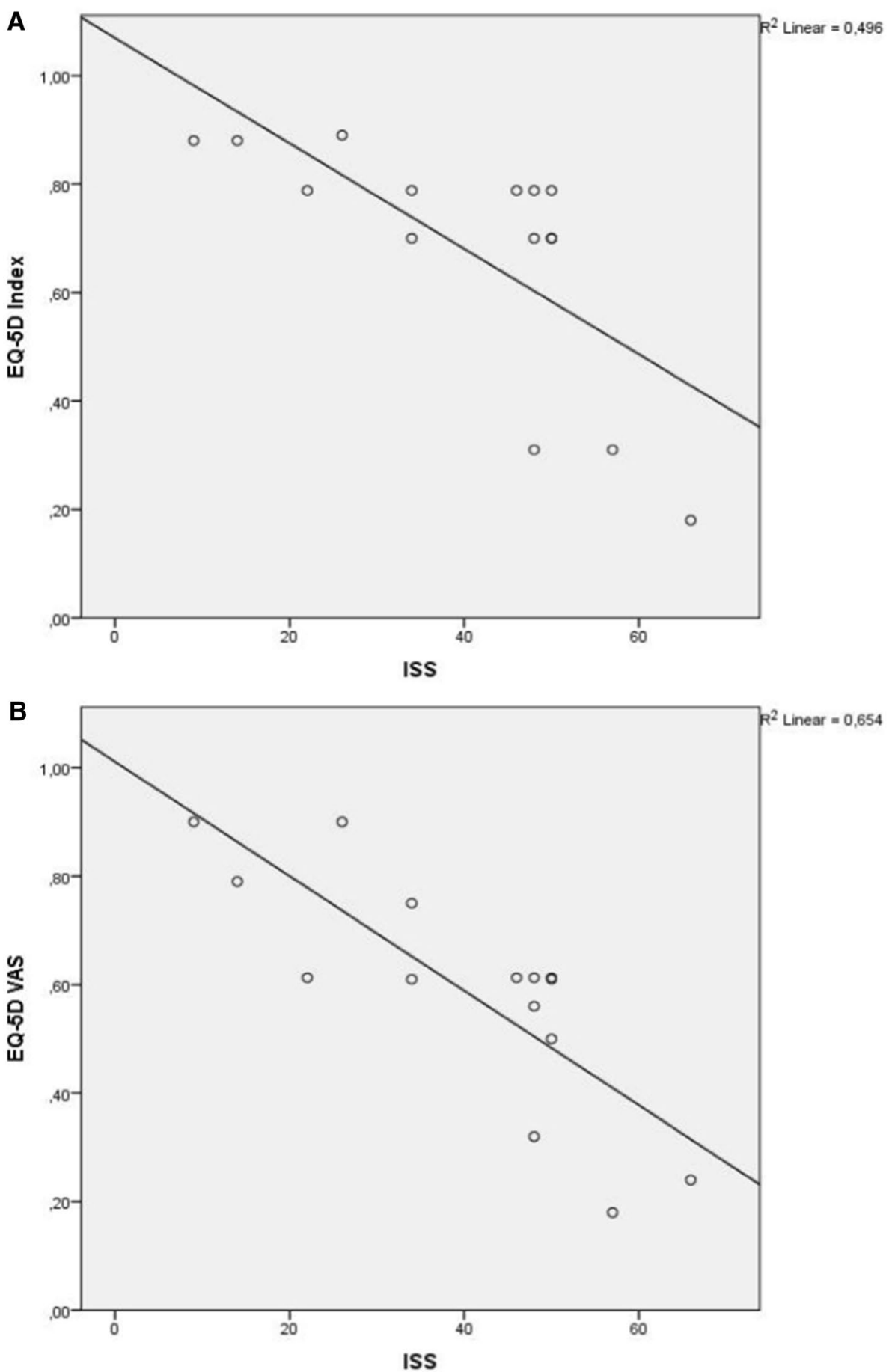

Supplementary Information The online version contains supplementary material available at https://doi.org/10.1007/s00586-021-07010-z.

Funding Open Access funding enabled and organized by Projekt DEAL. No funding.

\section{Declarations}

Conflict of interest None of the authors have any conflicts of interest related to this work.
Ethical approval Ethical approval was obtained from the Human Research Ethics Committee of the University of Munich, LMU, Germany (No. 18-745).

Open Access This article is licensed under a Creative Commons Attribution 4.0 International License, which permits use, sharing, adaptation, distribution and reproduction in any medium or format, as long as you give appropriate credit to the original author(s) and the source, provide a link to the Creative Commons licence, and indicate if changes were made. The images or other third party material in this article are 
included in the article's Creative Commons licence, unless indicated otherwise in a credit line to the material. If material is not included in the article's Creative Commons licence and your intended use is not permitted by statutory regulation or exceeds the permitted use, you will need to obtain permission directly from the copyright holder. To view a copy of this licence, visit http://creativecommons.org/licenses/by/4.0/.

\section{References}

1. Roy-Camille R, Saillant G, Gagna G, Mazel C (1985) Transverse fracture of the upper sacrum: suicidal jumper's fracture. Spine (Phila Pa 1976). https://doi.org/10.1097/00007632-19851 1000-00011

2. Piltz S, Rubenbauer B, Böcker W, Trentzsch H (2018) Reduction and fixation of displaced $\mathrm{U}$-shaped sacral fractures using lumbopelvic fixation: technical recommendations. Eur Spine J 27:3025-3033. https://doi.org/10.1007/s00586-017-5368-0

3. Starantzis KA, Mirzashahi B, Behrbalk E et al (2014) Open reduction and posterior instrumentation of type 3 high transverse sacral fracture-dislocation: technical note. J Neurosurg Spine 21:286291. https://doi.org/10.3171/2014.4.SPINE13835

4. Mameghani AT, Jeanneret B, Schären S (2016) Ilio-sacral stabilization of sacral jumper's fractures using the AO-Spine fracture system: a novel surgical technique. Glob Spine J 6:s-00361582727. https://doi.org/10.1055/s-0036-1582727

5. Lindahl J, Mäkinen TJ, Koskinen SK, Söderlund T (2014) Factors associated with outcome of spinopelvic dissociation treated with lumbopelvic fixation. Injury 45:1914-1920. https://doi.org/ 10.1016/j.injury.2014.09.003

6. Allen BL Jr, Ferguson RL (1982) The Galveston technique for L rod instrumentation of the scoliotic spine. Spine.pdf

7. Bellabarba C, Schildhauer TA, Vaccaro AR, Chapman JR (2006) Complications associated with surgical stabilization of high-grade sacral fracture dislocations with spino-pelvic instability. Spine 31:80-88. https://doi.org/10.1097/01.brs.0000217949.31762.be

8. Hessmann M, Degreif J, Mayer A et al (2000) Transverse sacral fracture with intrapelvic intrusion of the lumbosacral spine: case report and review of the literature. J Trauma: Inj Infect Crit Care 49:754-757. https://doi.org/10.1097/00005373-200010000-00027

9. Kellam JF, McMurtry RY, Paley D, Tile M (1987) The unstable pelvic fracture. Operative treatment. Orthop Clin North Am 18:25-41

10. Pascal-Moussellard H, Hirsch C, Bonaccorsi R (2016) Osteosynthesis in sacral fracture and lumbosacral dislocation. Orthop Traumatol Surg Res 102:S45-S57. https://doi.org/10.1016/j.otsr. 2015.12.002

11. Rhee W-T, You S-H, Jang Y-G, Lee S-Y (2007) Lumbo-sacropelvic fixation using iliac screws for the complex lumbo-sacral fractures. J Korean Neurosurg Soc 42:495. https://doi.org/10. 3340/jkns.2007.42.6.495

12. Cecchinato R, Redaelli A, Martini C et al (2017) Long fusions to $\mathrm{S} 1$ with or without pelvic fixation can induce relevant acute variations in pelvic incidence: a retrospective cohort study of adult spine deformity surgery. Eur Spine J 26:436-441. https://doi.org/ 10.1007/s00586-017-5154-z

13. Hsieh PC, Ondra SL, Wienecke RJ et al (2007) A novel approach to sagittal balance restoration following iatrogenic sacral fracture and resulting sacral kyphotic deformity: technical note. J Neurosurg Spine 6:368-372. https://doi.org/10.3171/spi.2007.6.4.15

14. Gromov K, Troelsen A, Raaschou S, et al (2019) Tissue adhesive for wound closure reduces immediate postoperative wound dressing changes after primary TKA: a randomized controlled study in simultaneous bilateral TKA. In: Clinical orthopaedics and related research

15. Kammerlander C, Pfeufer D, Lisitano LA et al (2018) Inability of older adult patients with hip fracture to maintain postoperative weight-bearing restrictions. J Bone Jt Surg: Am. https://doi.org/ 10.2106/JBJS.17.01222

16. Pfeufer D, Zeller A, Mehaffey S et al (2019) Weight-bearing restrictions reduce postoperative mobility in elderly hip fracture patients. Arch Orthop Trauma Surg. https://doi.org/10.1007/ s00402-019-03193-9

Publisher's Note Springer Nature remains neutral with regard to jurisdictional claims in published maps and institutional affiliations. 